Tohoku J. Exp. Med., 2010, 222, 97-104

\title{
The Pre-Supplementary and Primary Motor Areas Generate Rhythm for Voluntary Eye Opening and Closing Movements
}

\author{
Yukihisa Suzuki, ${ }^{1,2}$ Motohiro Kiyosawa, ${ }^{1,2}$ Manabu Mochizuki, ${ }^{1}$ Kiichi Ishiwata ${ }^{2}$ \\ and Kenji Ishii ${ }^{2}$ \\ ${ }^{1}$ Department of Ophthalmology and Visual Science, Tokyo Medical and Dental University Graduate School, \\ Tokyo, Japan \\ ${ }^{2}$ Positron Medical Center, Tokyo Metropolitan Institute of Gerontology, Tokyo, Japan
}

\begin{abstract}
Blinking and opening/closing of the eyelid are considered to be different movements with independent control mechanisms. Apraxia of lid opening (ALO) is a clinical syndrome in which patients experience difficulty in opening their eyes voluntarily. Our previous study with fluorodeoxyglucose and positron emission tomography (PET) has suggested that functional impairments in the supplementary motor area (SMA) and the anterior cingulate gyrus may be involved in the pathophysiology of ALO. The aim of this study was to explore the physiological mechanisms for voluntary eyelid opening/closing and the difference between self-initiated and triggered movements, using $\left[{ }^{15} \mathrm{O}\right] \mathrm{H}_{2} \mathrm{O}$ and PET. We measured the regional cerebral blood flow in 8 healthy subjects under 3 conditions: [A] at rest with eyes closed, [B] with self-paced lid opening/closing, and [C] with triggered lid opening/closing. All tasks were done with a blindfold to exclude the influence of visual input. The SMA proper and the angular gyrus were activated during self-paced and triggered lid opening/closing movements; however, the pre-SMA and the primary motor area (M1) were activated only during self-paced movements. The anterior cingulate gyrus and the cerebellum were activated during self-paced condition over triggered condition. The roles of SMA, M1 and cerebellum were assumed in eyelid opening/closing movements: the preparation and processing of movements in SMA, execution of movements in M1, and rhythmic generation in pre-SMA, M1 and cerebellum. We suggest that the activation in pre-SMA, anterior cingulate gyrus, and cerebellum may be responsible for the self-initiated eyelid opening/closing movements.
\end{abstract}

Keywords: anterior cingulate gyrus; eye opening and closing; positron emission tomography; regional cerebral blood flow; supplementary motor area

Tohoku J. Exp. Med., 2010, 222 (2), 97-104. 으 2010 Tohoku University Medical Press

Blinking and opening/closing of the eyelid are considered to be different movements with independent control mechanisms. Apraxia of lid opening (ALO) is a clinical syndrome in which patients experience difficulty in voluntarily opening their eyes (Kerty and Eidal 2006). It is thought that supranuclear disorder is related to the etiology of ALO, and it has been reported that ALO accompanies blepharospasm (Suzuki et al. 2003), Parkinson's disease (Fuss et al. 2004), Shy-Drager syndrome, and progressive supranuclear palsy (Piccione et al. 1997). Botulinum toxin treatment is not often effective, but surgical treatments, such as blepharoplasty, limited myectomy, aponeurosis repair, and frontalis suspension are sometimes effective (Kerty and Eidal 2006). In our previous positron emission tomography (PET) study, cerebral glucose hypometabolism was observed in the supplementary motor area (SMA) and anterior cingulate gyrus in patients with ALO during an awake resting condition with their eyes closed. Based on these results, we concluded that in ALO patients, the mechanism for initiating voluntary lid opening is impaired (Suzuki et al. 2003).

The central control mechanisms for voluntary movements in various parts of the body have been extensively studied using functional neuroimaging techniques for different modes of movements in terms of frequency (Sadato et al. 1996; Kawashima et al. 1999; Tankus et al. 2009), complexity (Avanzino et al. 2008), and presence or absence of external cues (Shima et al. 1991; Wessel et al. 1997; Deiber et al. 1999; Cunnington et al. 2002). However, only a few neuroimaging studies have been conducted on blinking (Van Eimeren et al. 2001), and there is no report on the functional neuroimaging of eye opening and closing. We believe that the cortical regions responsible for voluntary lid opening/ closing might not be the same as those responsible for

Received July 5, 2010; revision accepted for publication August 30, 2010. doi: 10.1620/tjem.222.97

Correspondence: Yukihisa Suzuki M.D., Ph.D., Department of Ophthalmology and Visual Science, Tokyo Medical and Dental University,

Graduated School, Yushima 1 chome-5-45, Bunkyo-ku, Tokyo, 113-8519 Japan.

e-mail: suzuki@f8.dion.ne.jp 
blinking.

A number of studies have shown that the SMA plays an important role in the preparation of voluntary movements in the hands, foot, face, and other parts of the body (Yamamoto et al. 2004). A previous PET study has shown that the right primary motor cortex (M1) and SMA are activated during blinking (Van Eimeren et al. 2001); however, the central control mechanism for voluntary lid opening has not yet been elucidated. Even in the case of similar hand or finger movements, it has been demonstrated that changes in cerebral blood flow (CBF) were different for self-initiated movements and externally cued movements (Wessel et al. 1997; Deiber et al. 1999). ALO patients can open their eyes in response to a light touch on the upper lids (a sensory trick), although they find it difficult to open their eyes voluntarily. Thus, we have investigated CBF responses to both self-initiated and externally-cued lid movements. In the present study, we conducted a PET study, instead of functional MRI (fMRI) study. PET has several technical advantages over fMRI for the purpose of exploring CNS motor control functions. The amount of the signal change is much larger in PET, which can compensate for small sample numbers. Furthermore, the sensitivity of detecting signal changes in PET is homogenous throughout the brain based on the quality control of quantitative data acquisition.

Here, we hypothesize that the brain areas are responsible for the etiology of ALO, such as the SMA and the anterior cingulate gyrus, are related to voluntary lid opening. We, therefore, conducted a PET-activation study on voluntary lid opening/closing movements, but not on blinking movements.

\section{Materials and Methods}

We studied 8 right-handed healthy volunteers (4 men and 4 women; age range, 20-24 years). The protocol for this study was approved by the Institutional Ethics Committee of the Tokyo Metropolitan Institute of Gerontology, and signed informed consent was obtained from each subject after the procedures were explained.

\section{Positron Emission Tomography Data Acquisition}

PET scans were obtained with the HEADTOME-V SET 2400W scanner (Shimadzu, Kyoto, Japan) at the Positron Medical Center, Tokyo Metropolitan Institute of Gerontology. The attenuation was corrected by a transmission scan with a ${ }^{68} \mathrm{Ga} /{ }^{68} \mathrm{Ge}$ rotating source before the administration of $\left[{ }^{15} \mathrm{O}\right] \mathrm{H}_{2} \mathrm{O}$. A bolus of $150 \mathrm{MBq}\left[{ }^{15} \mathrm{O}\right] \mathrm{H}_{2} \mathrm{O}$ was injected intravenously, and a 1-min emission scan was initiated after radioactivity was observed in the brain. No arterial blood sampling was performed, and thus, the images obtained were considered to represent tissue activity. Regional tissue activity measured with short scanning times of 40-60 s correlated almost linearly with regional CBF (rCBF) (Fox and Mintun 1989). Thus, the regional radioactivity was regarded as a measure of $\mathrm{rCBF}$ for further analysis.

The scanning room was dimly lit, and all the subjects were blindfolded with an eye mask throughout the PET study. Opening/ closing of the lids and eye movements were monitored by electrooculography (EOG) (Fig. 1). We used a blindfold that did not exert pressure on the eyes or eyelids, and thus did not interfere with lid opening/ closing; we also used EOG electrodes that did not cause excessive stimulation or pressure. We recorded the condition of the subjects during the scan using a video camera and confirmed that there were no unnecessary facial or body movements.

\section{Magnetic Resonance Imaging}

Magnetic resonance imaging (MRI) scans were obtained for all the subjects with a 1.5-Tesla scanner Signa Horizon (General Electric, Milwaukee, WI) to rule out organic brain disorders, and for further image processing. Transaxial images with $\mathrm{T} 1$-weighted contrast (3DSPGR, TR $=400 \mathrm{~ms}$; TE $=9 \mathrm{~ms}$; matrix size $=256 \times 256 \times 124$; voxel size $=0.94 \times 0.94 \times 1.3 \mathrm{~mm}$ ) and T2-weighted contrast (First Spin Echo, TR $=3000 \mathrm{~ms}$; TE $=100 \mathrm{~ms}$; matrix size $=256 \times 256 \times$ 20 ; voxel size $=0.7 \times 0.7 \times 6.5 \mathrm{~mm}$ ) were obtained.

\section{Task Design}

Scans were obtained under 3 conditions, and 2 scans were obtained for each condition: [A] at rest with eyes closed (rest), [B] with opening and closing of the lids controlled by the subject (selfpaced lid opening/closing), and [C] with opening and closing of the lids in response to sound stimuli (triggered lid opening/closing). All the tasks were started $30 \mathrm{~s}$ before the PET scan.

Rest. The subjects lay on the scanner bed in the supine position with their eyes closed and were required to keep their eyelids and other body parts still. They were requested to avoid thinking of any mental image of movement or any other sensory perception during the scans.

Self-paced lid opening/closing. Before the PET scan, subjects had a practice session so that they could maintain the frequency of self-paced lid opening and closing at approximately $0.2 \mathrm{~Hz}$. This frequency was chosen because most of the subjects could comfortably perform the movements at this frequency. The subjects were instructed to open their eyes and then close them immediately. They were instructed to keep the movements smooth and prompt, without additional movement of any other part of the face or body. Their eyes were closed in the interval between the movements.

Triggered lid opening/closing. The subjects were instructed to open their eyes upon hearing a sound produced by a personal computer (PC), close them immediately thereafter, and keep them closed until the sound was heard again. The timing of the self-paced lid opening/ closing was recorded with an EOG, using a PC-controlled device. To generate the sounds for triggering the eye movements, a time series from the self-paced lid opening/closing task performed by the subject was used for the same subject, after adding a randomized deviation of up to $20 \%$ of the average interval. The timing of the self-paced movements were regenerated not only to keep the number of movements during the scan equal for the triggered movement tasks but also to prevent subjects from anticipating the timing of the sound.

\section{Data Processing and Statistical Analyses}

All the image processing and statistical analyses unless otherwise indicated were performed using statistical parametric mapping (SPM 2; Welcome Department of Cognitive Neurology, London, UK) implemented in Matlab version 7.1 (Mathworks Inc., Sherborn, MA) (Friston et al. 1991). The scans from each subject were realigned using the least squares approach to remove the effect of head motion. After realignment, all the PET images were registered three-dimensionally to the individual 3D MR images with the Automatic Multimodality Images Registration (AMIR) program (Ardekani et al. 

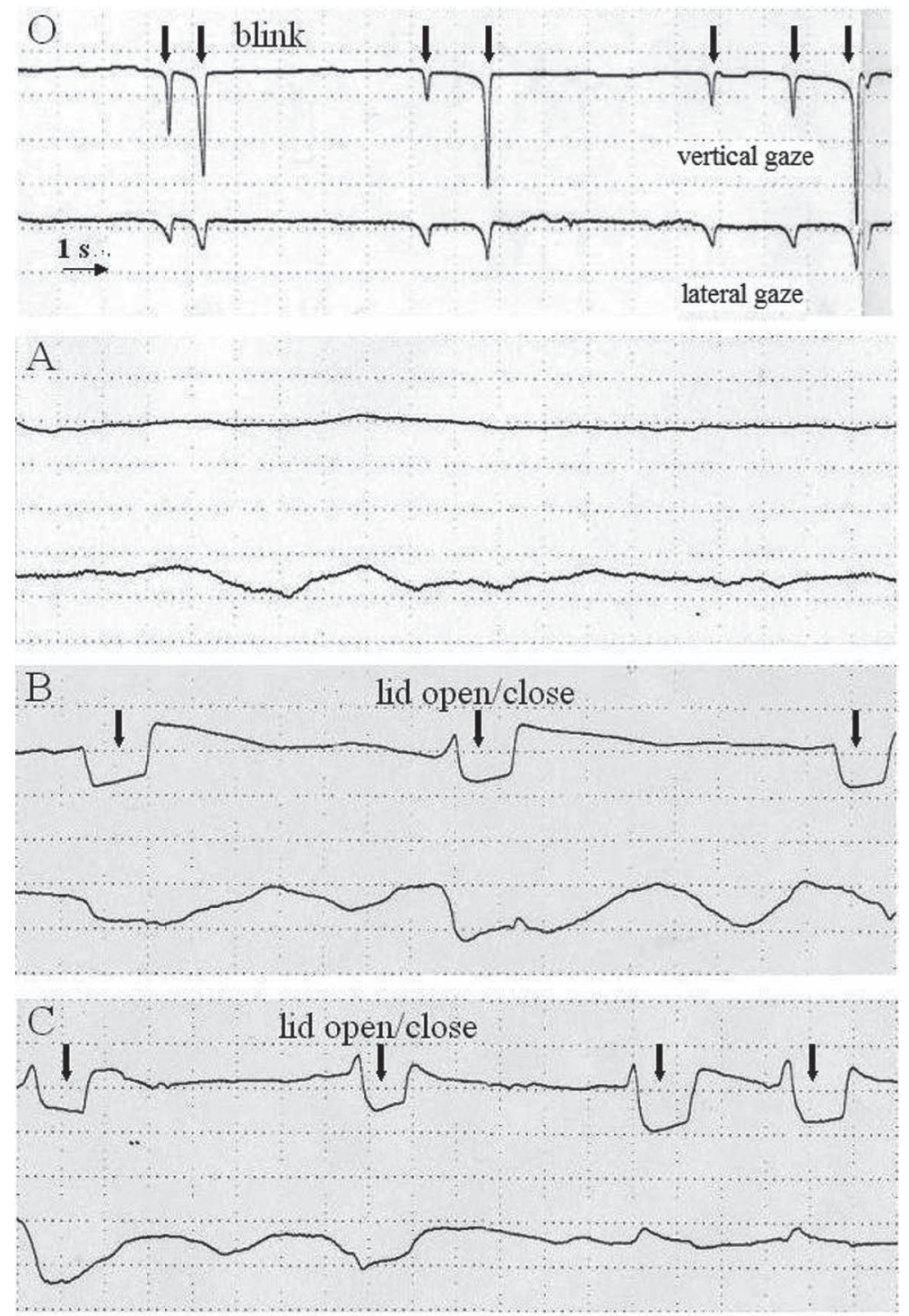

Fig. 1. Electrooculogram during each condition.

Electrooculographic recordings were conducted before the study $(\mathrm{O})$ and during each condition $(\mathrm{A}-\mathrm{C})$. The upper line represents the vertical gaze and the lower line represents the lateral gaze.

(O) At rest with eyes open. Reflex blinking was recorded every few seconds.

(A) At rest: at rest with eyes closed. There were no major eye movements or blinking.

(B) Self-paced lid opening/closing: subjects timed the opening and closing of their eyes.

(C) Triggered lid opening/closing: subjects opened and closed their eyes in response to certain tones.

1995). Using a template produced by the Montreal Neurological Institute (MNI), the MR images were spatially normalized to a standard anatomical space (the MNI space) (Talairach and Tournoux 1988). All PET images were normalized using the transformation matrix of the co-registered MRI and smoothed with a Gaussian kernel of $10 \mathrm{~mm}$ full-width half-maximum in the $\mathrm{x}, \mathrm{y}$, and $\mathrm{z}$ axes.

After the appropriate design matrix was specified, the condition effects were estimated according to the general linear model at each voxel. The design matrix included global activity as a confounding covariate; this analysis can therefore be regarded as an ANCOVA (multi-subject PET model with covariate or similar) (Friston et al. 1990). First, to investigate the brain regions related to each condition, a contrast image of (1) self-paced lid opening/closing minus rest and
(2) triggered lid opening/closing minus rest were examined. To examine the difference between self-paced and triggered movements, contrast images of (3) self-paced lid opening/closing minus triggered lid opening/closing were used. In the same way, contrast images of (4) triggered lid opening/closing minus self-paced lid opening/closing were examined. For all regions reported as being activated, the level of significance exceeded $P<0.05$, false discovery rate (FDR)corrected with an extent threshold of 30 voxels to exclude small areas showing changes in rCBF. The resulting set of voxel values for each contrast image was used to construct a statistical parametric map of the $t$ statistic SPM (t). The significance of each region was estimated using distributional approximations from the theory of Gaussian fields (Worsley et al. 1999). 
We used a direct comparison of homologous with the flipped images in the right-left direction to determine if there was any lateralization in the activity of the M1 or other areas during each task (Mizoguchi et al. 2005; Callan et al. 2006). We created flipped images of all the PET images and subtracted the flipped contrast images (flipped task image minus the flipped rest image) and non-flipped contrast images (non-flipped task image minus the non-flipped rest image). The subtracted self-paced lid opening/closing image was a contrast of [self-paced lid opening/closing minus rest] minus [flipped self-paced lid opening/closing minus flipped rest]. We used the same analysis to the contrasts of triggered lid opening/closing minus rest. The level of significance exceeded $P<0.05$, FDR-corrected with an extent threshold of 30 voxels to exclude small areas showing a change in $\mathrm{rCBF}$.

\section{Results}

Activation under Basic Conditions

Self-paced lid opening/closing vs rest. Self-paced lid opening/closing resulted in a significant increase in relative rCBF over rCBF values of subjects at rest in the SMA proper, pre-SMA, right angular gyrus, and left M1 (Fig. 2A). The $\mathrm{Z}$ score and cluster size of the relative activation areas were 4.26 and 193 (SMA proper), 3.99 and 97 (pre-SMA), 3.80 and 59 (right angular gyrus), and 3.63 and 81 (left M1), respectively (Table 1).

Triggered lid opening/closing vs rest. An increase in rCBF was observed in the SMA proper, right superior temporal gyrus, right insular cortex, and left angular gyrus of triggered lid opening/closing subjects vs. those at rest (Fig. 2B). The $\mathrm{Z}$ score and cluster size were 4.08 and 147 (SMA proper), 3.87 and 75 (right superior temporal gyrus), 3.79 and 77 (right insular cortex), and 3.58 and 43 (left angular gyrus), respectively (Table 1).

\section{Comparison between Two Motor Conditions}

Self-paced lid opening/closing vs triggered lid opening/closing. A higher $\mathrm{rCBF}$ was observed in the anterior cingulate gyrus, cerebellar vermis, and left cerebellar hemisphere (Fig. 3A). The Z score and cluster size of the relative activation areas were 3.78 and 33 (anterior cingulate gyrus), 3.57 and 31 (cerebellar vermis), and 3.56 and 40 (left cerebellar hemisphere), respectively (Table 1).

Triggered lid opening/closing vs self-paced lid opening/closing. A higher $\mathrm{rCBF}$ was observed in the right inferior frontal gyrus, posterior cingulate gyrus, and SMA proper (Fig. 3B). The $\mathrm{Z}$ score and cluster size were 4.18 and 111 (right inferior frontal gyrus), 4.06 and 277 (posterior cingulate gyrus), and 3.53 and 94 (SMA proper), respectively (Table 1).

\section{Subtraction between Non-flipped Images and Flipped Images}

There was no significant relative activation in the M1 or other areas under subtraction image during self-paced task. In the same way, there was no activation in the M1 or other areas during triggered task.

\section{Discussion}

The aim of our study was to explore the physiological mechanism of eyelid opening/closing and the difference between self-initiated and triggered movements. We observed that the SMA proper was activated during both tasks, and that the pre-SMA was activated during self-paced lid opening/closing. A comparison of the self-paced and triggered tasks demonstrated a relative increase in $\mathrm{rCBF}$ in the anterior cingulate gyrus and cerebellum.

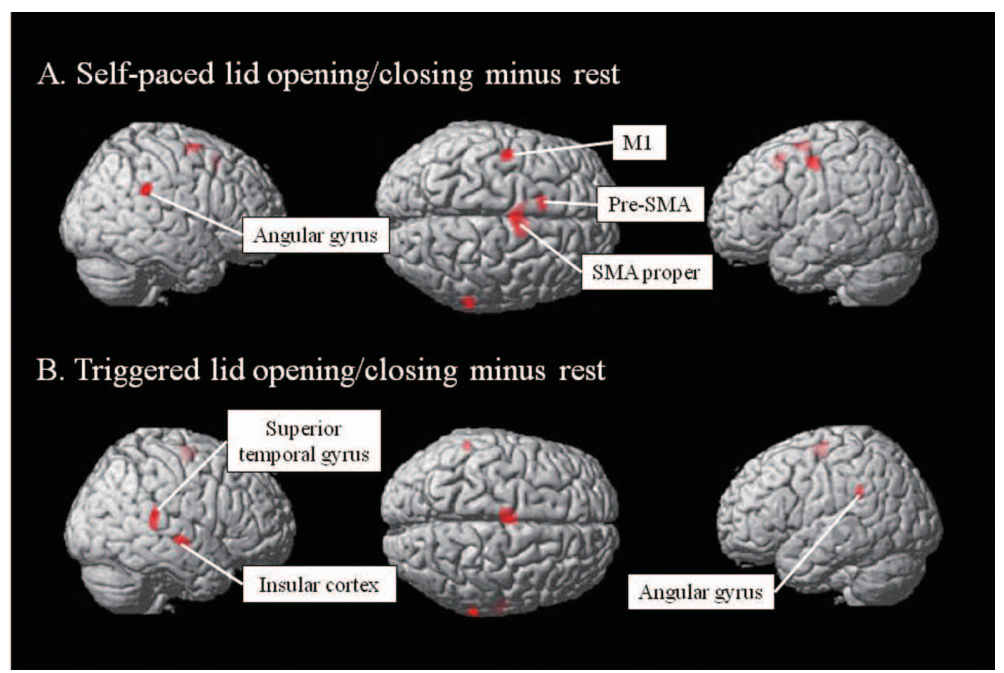

Fig. 2. Activation areas during self-paced lid opening/closing and triggered lid opening/closing.

Lateral and superior views of a statistical parametric map (SPM) rendered into standard stereotactic space $(P<0.05$, FDR-corrected).

(A) Self-paced lid opening/closing minus rest. (B) Triggered lid opening/closing minus rest. The supplementary motor area (SMA) proper was activated during both tasks. The pre-SMA and left primary motor area (M1) were activated during self-paced eye opening/closing.

Extent threshold, $\mathrm{k}=30$ voxels. 
Table 1. Areas and coordinates for the maxima of increases in regional cerebral blood flow.

\begin{tabular}{lccccc}
\hline \multicolumn{1}{c}{ Area activated } & $\mathrm{x}$ & $\mathrm{y}$ & $\mathrm{z}$ & $\mathrm{Z}$ score & cluster size \\
\hline Self-paced lid opening/closing vs. rest & & & & & \\
SMA proper & 10 & 2 & 64 & 4.26 & 193 \\
Pre-SMA & -10 & 18 & 50 & 3.99 & 97 \\
Angular gyrus (R) & 64 & -38 & 30 & 3.80 & 59 \\
M1 (L) & -44 & -12 & 50 & 3.63 & 81 \\
\hline Triggered lid opening/closing vs. rest & & & & \\
SMA proper & -8 & -12 & 68 & 4.08 & 147 \\
Superior temporal gyrus (R) & 68 & -38 & 10 & 3.87 & 75 \\
Insular cortex (R) & 64 & -16 & -4 & 3.79 & 77 \\
Angular gyrus (L) & -58 & -42 & 28 & 3.58 & 43 \\
\hline Self-paced lid opening/closing vs. triggered lid opening/closing & & & \\
Anterior cingulate & -2 & 26 & 16 & 3.78 & 33 \\
Cerebellar vermis & 0 & -38 & -24 & 3.57 & 31 \\
Cerebellar hemisphere (L) & -14 & -78 & -26 & 3.56 & 40 \\
\hline Triggered lid opening/closing vs. self-paced lid opening/closing & & & \\
Inferior frontal gyrus (R) & 58 & 36 & 16 & 4.18 & 111 \\
Posterior cingulate & 6 & -30 & 36 & 4.06 & 277 \\
SMA proper & -12 & -20 & 66 & 3.53 & 94 \\
\hline
\end{tabular}

Areas with $\mathrm{Z}>3.25(P<0.05$, FDR-corrected $)$ are listed here.

Global $\mathrm{CBF}=50$

SMA, supplementary motor area; M1, primary motor area

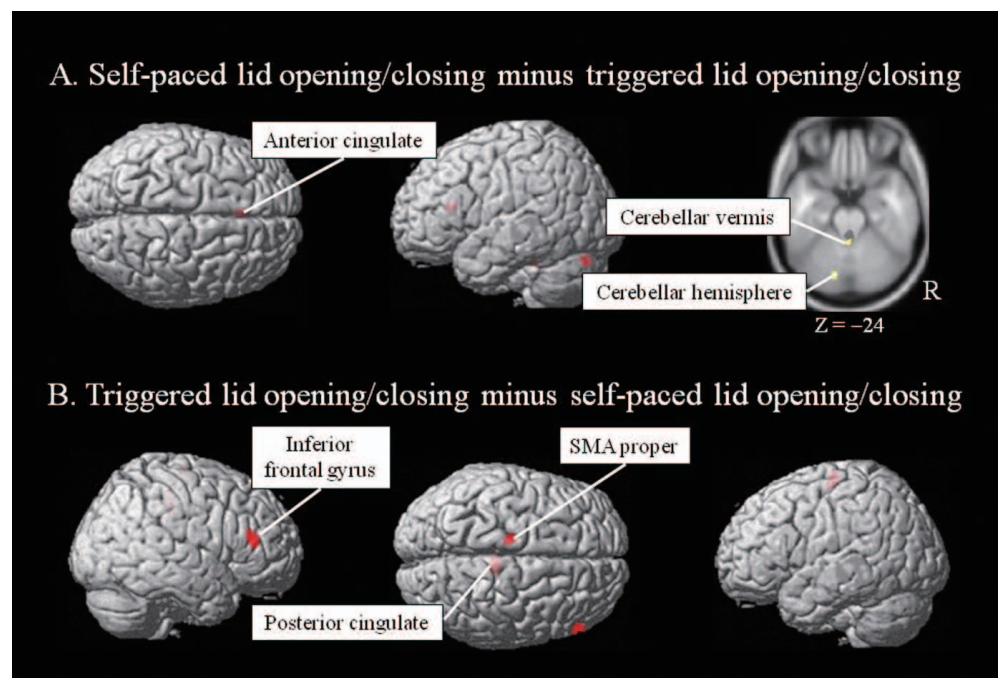

Fig. 3. Relative activation areas between self-paced lid opening/closing and triggered lid opening/closing.

Lateral and superior views of a statistical parametric map (SPM) rendered into standard stereotactic space, and one slice of axial image (A) $(P<0.05$, FDR-corrected).

(A) Self-paced lid opening/closing minus triggered lid opening/closing. Relative activation in the anterior cingulate gyrus and the cerebellum was observed. (B) Triggered lid opening/closing minus self-paced lid opening/closing. Relative activation in the inferior frontal gyrus, posterior cingulate gyrus, and supplementary motor area (SMA) proper was observed.

Extent threshold, $\mathrm{k}=30$ voxels.

\section{SMA Proper and Pre-SMA}

The SMA proper, which is located posterior to the vertical line transversing the anterior commissure (VCA line), and the pre-SMA, which is located anterior to the VCA line (Yamamoto et al. 2004), belong to the medial part of the premotor cortex. They play a major role in the preparation 
and processing of voluntary movements (Peck et al. 2009).

We observed that the SMA proper was activated during both the self-paced and triggered tasks, while the pre-SMA was activated during self-paced lid opening/closing but not during triggered lid opening/closing. A previous study observed that the SMA proper and pre-SMA were activated during self-paced and metronome-paced finger movements, but the pre-SMA was more active during self-paced movements (Wessel et al. 1997). These results may indicate that the pre-SMA is more closely related to self-initiated movements than the SMA proper.

\section{Anterior Cingulate Gyrus}

Movements of the lids during self-paced lid opening/ closing were the same as those during triggered lid opening/ closing. A comparison of the self-paced lid opening/closing and triggered lid opening/closing tasks demonstrated a relative increase in $\mathrm{rCBF}$ in the anterior cingulate gyrus. Shima et al. (1991) reported that the anterior cingulate gyrus is involved in the initial motor preparation for voluntary movements. The activation of the pre-SMA and anterior cingulate gyrus has been shown to be greater in the case of selfgenerated movements than in the case of externally determined finger movements (Wessel et al. 1997; Deiber et al. 1999; Cunnington et al. 2002). On the basis of these findings, we believe that the anterior cingulate gyrus plays a role in the preparation of voluntary movements in coordination with the pre-SMA and plays a greater role in self-generated movements.

\section{Primary Motor Area}

A previous study reported that the right-lateralized motor cortex was activated during volitional blinking (Van Eimeren et al. 2001). In the present study, the left M1 was activated during self-paced lid opening/closing, but, there was no significant difference between right and left M1 activation in the subtract analysis using flipped images. Generally, the superior part of face muscles is controlled by the bilateral central nerve (Brodal 1981). Therefore, we suggested that the bilateral M1 was activated during lid movement. The M1 activation may sometimes show significance unilaterally using SPM software because of the threshold effect. In the present study, the M1 was activated during the self-paced task but not during the triggered task. However, Van Eimeren et al. (2001) reported activation in the M1 during both volitional and triggered blinking. We attempted to examine the M1 activation during self-paced and triggered tasks in each subject. Images of the "selfpaced task vs. rest" and "triggered task vs. rest" in each subject were made, and we examined whether activation in the M1 was statistically significant, i.e., with a level of significance exceeding $P<0.01$ ( $\mathrm{Z}>2.80$, uncorrected) using SPM. We observed activation in the M1 in all subjects during the self-paced task and in 4 subjects during the triggered task. We suspected that activation in the M1 during the triggered task was not exceeded significant level using the SPM program.

\section{Angular Gyrus}

We observed that the angular gyrus was activated during both the self-paced and triggered tasks. Mort et al. observed relative activation in the angular gyrus under the contrast of reflexive saccade minus voluntary saccade using fMRI (Mort et al. 2003). Shirani et al. (2009) studied patients with hemispatial neglect after infarction, and they reported hypoperfusion in the angular gyrus. The function of the angular gyrus is uncertain; however, it seems to play a role in the awareness and exploration of space.

\section{Cerebellum}

We observed relative activations in the cerebellar hemisphere and vermis between the self-paced and triggered tasks. Several previous studies have reported that the cerebellar vermis and cerebellar hemisphere were activated during horizontal eye movement (Stephan et al. 2002; Hüfner et al. 2008) and blinking (Van Eimeren et al. 2001; Dimitrova et al. 2002). According to these reports, the area that was activated by horizontal eye movement almost overlapped with the area activated by lid movement.

\section{Difference between Self-paced Movement and Triggered Movement}

We observed that the pre-SMA was activated during self-paced lid opening/closing but not during triggered lid opening/closing. A relative increase in $\mathrm{rCBF}$ in the anterior cingulate gyrus might be one of the characteristic differences between the self-paced and triggered tasks. We also observed activation of the cerebellar hemisphere and vermis under this contrast. When we try to move our lid by selfpaced, we should produce rhythm by ourselves. Previous studies have reported that the M1, primary somatosensory cortex, SMA, premotor cortex, and cerebellar hemisphere are related to the self generation of rhythmic movements (Gerwig et al. 2007; Thaut et al. 2008). Moreover, Spencer et al. (2007) suggested that subregions of the cerebellum are selectively engaged during tasks involving event timing.

\section{Difference between Lid Opening/closing and Blinking}

Blinking is a movement that involves closing of the eyelid followed by its opening, while eyelid opening/closing that is a discontinuous movement of lid opening and closing and begins with the lid close. Blinking is classified into volitional, spontaneous and reflex. During each type of blinking, the neural control is distributed on partially overlapping circuitries in cortical, subcortical, and brainstem structures (Bour et al. 2000). Reflex blinking is involuntary blinking with stimulus such as light, touch or wind. Spontaneous blinks seem to arise from a central "blink generator" that can be influenced by several factors, such as visual and cognitive task requirements, and emotion. Neurobiological studies have identified the cerebellum and its brainstem connections as essential components of the 
eyeblink conditioning circuit (Gerwig et al. 2007). Other forebrain areas, including basal ganglia, neocortex, and septal nuclei, have been less extensively studied but may also play a role in eyeblink conditioning. A previous study reported that the SMA proper, pre-SMA, prefrontal cortex, and M1 were activated during volitional blinking (Van Eimeren et al. 2001), and our result of lid opening/closing are consistent with these data. Thus, the areas in the brain that are associated with volitional blinking and volitional eyelid opening/closing might share the same neural substrates. However, there is an independent feature in the central mechanism of spontaneous blinking.

\section{Understanding the Pathophysiology of ALO}

It is difficult for ALO patients to open their eyelids voluntarily, but once opened, they can keep them open without difficulty (Kerty and Eidal 2006). This suggests that in ALO patients, only the initiation of lid opening may be impaired. In our previous PET study, cerebral glucose hypometabolism was observed in the SMA proper and anterior cingulate gyrus in ALO patients (Suzuki et al. 2003). ALO patients can sometimes open their eyes in response to stimuli applied to their eyelids (a sensory trick). In the present study, a comparison of the self-paced lid opening/closing and triggered lid opening/closing tasks demonstrated that the anterior cingulate gyrus played a selective role in the self-initiation of lid movement. The results of the current study suggest that the pre-SMA and anterior cingulate gyrus are related to the pathophysiology of ALO.

\section{Conclusions}

The SMA proper, pre-SMA and other areas in the brain are related to the control of lid opening/closing. The preSMA, anterior cingulate gyrus, and cerebellum may contribute to self-initiated movement, and these areas might be related to the etiology of ALO.

\section{Acknowledgments}

This research was supported by Grants-in-Aid for Scientific Research (B) 21791725 (Y.S.) from the Japan Society for the Promotion of Science.

We are grateful to Dr. Shoichi Mizoguchi, Dr. Michio Senda and Dr. Duco Hamasaki for their useful comments and discussions regarding the manuscript. We would also like to thank Dr. Kazunori Kawamura, Dr. Keiichi Oda, Ms Miyoko Ando, and Dr. Yuichi Kimura for their technical assistance with the PET study.

\section{References}

Ardekani, B.A., Braun, M., Hutton, B.F., Kanno, AI. \& Iida, H. (1995) A fully automatic multimodality image registration algorithm. J. Comput. Assist. Tomogr., 19, 615-623.

Avanzino, L., Bove, M., Trompetto, C., Tacchino, A., Ogliastro, C. \& Abbruzzese, G. (2008) 1-Hz repetitive TMS over ipsilateral motor cortex influences the performance of sequential finger movements of different complexity. Eur. J. Neurosci., 27, 1285-1291.

Bour, L.J., Aramideh, M. \& de Visser, B.W.O. (2000) Neurophysiological aspects of eye and eyelid movements during blinking in humans. J. Neurophysiol., 83, 166-176.

Brodal, A. (1981) third edition. Neurological anatomy. New York: Oxford university press. 495-499.

Callan, D.E., Tsytsarev, V., Hanakawa, T., Callan, A.M., Katsuhara, M., Fukuyama, H. \& Turner, R. (2006) Song and speech: brain regions involved with perception and covert production. Neuroimage, 31, 1327-1342.

Cunnington, R., Windischberger, C., Deecke, L. \& Moser, E. (2002) The preparation and execution of self-initiated and externallytriggered movement: a study of event-related fMRI. Neuroimage, 15, 373-385.

Deiber, M.-P., Honda, M., Ibanez, V., Sadato, N. \& Hallett, M. (1999) Mesial motor areas in self-initiated versus externally triggered movements examined with fMRI: effect of movement type and rate. J. Neurophysiol., 81, 3065-3077.

Dimitrova, A., Weber, J., Maschke, M., Elles, H.G., Kolb, F.P., Forsting, M., Diener, H.C. \& Timmann, D. (2002) Eyeblinkrelated areas in human cerebellum as shown by fMRI. Hum. Brain Mapp., 17, 100-115.

Fox, P.T. \& Mintun, M.A. (1989) Noninvasive functional brain mapping by change-distribution analysis of averaged PET images of $\mathrm{H}_{2}{ }^{15} \mathrm{O}$ tissue activity. J. Nucl. Med., 30, 141-149.

Fuss, G., Spiegel, J., Magnus, T., Moringlane, J.R., Becker, G. \& Dillmann, U. (2004) Improvement of apraxia of lid opening by STN-stimulation in a 70-year-old patient with Parkinson's disease. A case report. Minim. Invasive. Neurosurg., 47, 5860.

Friston, K.J., Frith, C.D., Liddle, P.F., Dolan, R.J., Lammertsma, A.A. \& Frackowiak, R.S.J. (1990) The relationship between global and local changes in PET scans. J. Cereb. Blood Flow Metab., 10, 458-466.

Friston, K.J., Frith, C.D., Liddle, P.F. \& Frackowiak, R.S. (1991) Comparing functional (PET) images: the assessment of significant change. J. Cereb. Blood Flow Metab., 11, 690-699.

Gerwig, M., Kolb, F.P. \& Timmann, D. (2007) The involvement of the human cerebellum in eyeblink conditioning. Cerebellum, 6, 38-57.

Hüfner, K., Stephan, T., Glasauer, S., Kalla, R., Riedel, E., Deutschländer, A., Dera, T., Wiesmann, M., Strupp, M. \& Brandt, T. (2008) Differences in saccade-evoked brain activation patterns with eyes open or eyes closed in complete darkness. Exp. Brain Res., 186, 419-430.

Kawashima, R., Inoue, K., Sugiura, M., Okada, K., Ogawa, A. \& Fukuda, H. (1999) A positron emission tomography study of self-paced finger movements at different frequencies. Neuroscience, 92, 107-112.

Kerty, E. \& Eidal, K. (2006) Apraxia of eyelid opening: clinical features and therapy. Eur. J. Ophthalmol., 16, 204-208.

Mizoguchi, S., Suzuki, Y., Kiyosawa, M., Mochizuki, M. \& Ishii, K. (2005) Differential activation of cerebral blood flow by stimulating amblyopic and fellow eye. Graefe's Arch. Clin. Exp. Ophthalmol., 243, 576-582.

Mort, D.J., Perry, R.J., Mannan, S.K., Hodgson, T.L., Anderson, E., Quest, R., McRobbie, D., McBride, A., Husain, M. \& Kennard, C. (2003) Differential cortical activation during voluntary and reflexive saccades in man. Neuroimage, 18, 231246.

Peck, K.K., Bradbury, M., Psaty, E.L., Brennan, N.P. \& Holodny, A.I. (2009) Joint activation of the supplementary motor area and presupplementary motor area during simultaneous motor and language functional MRI. Neuroreport, 20, 487-491.

Piccione, F., Mancini, E., Tonin, P. \& Bizzarini, M. (1997) Botulinum toxin treatment of apraxia of eyelid opening in progressive supranuclear palsy: report of two cases. Arch. Phys. Med. Rehabil., 78, 525-529.

Sadato, N., Ibanez, V., Deiber, M.P., Campbell, G., Leonardo, M. \& Hallett, M. (1996) Frequency-dependent changes of regional cerebral blood flow during finger movements. J. Cereb. Blood Flow Metab., 16, 23-33. 
Shima, K., Aya, K., Mushiake, H., Inase, M., Aizawa, H. \& Tanji, J. (1991) Two movement-related foci in the primate cingulate cortex observed in signal-triggered and self-paced forelimb movements. J. Neurophysiol., 65, 188-202.

Shirani, P., Thorn, J., Davis, C., Heidler-Gary, J., Newhart, M., Gottesman, R.F. \& Hillis, A.E. (2009) Severity of Hypoperfusion in Distinct Brain Regions Predicts Severity of Hemispatial Neglect in Different Reference Frames. Stroke, 40, 35633566.

Spencer, R.M., Verstynen, T., Brett, M. \& Ivry, R. (2007) Cerebellar activation during discrete and not continuous timed movements: an fMRI study. Neuroimage, 36, 378-387.

Suzuki, Y., Kiyosawa, M., Ohno, N., Mochizuki, M., Inaba, A., Mizusawa, H., Ishii, K. \& Senda, M. (2003) Glucose hypometabolism in medial frontal cortex of patients with apraxia of lid opening. Graefe's Arch. Clin. Exp. Ophthalmol., 241, 529534.

Stephan, T., Mascolo, A., Yousry, T.A., Bense, S., Brandt, T. \& Dieterich, M. (2002) Changes in cerebellar activation pattern during two successive sequences of saccades. Hum. Brain Mapp., 16, 63-70.

Tankus, A., Yeshurun, Y., Flash, T. \& Fried, I. (2009) Encoding of speed and direction of movement in the human supplementary motor area. J. Neurosurg., 110, 1304-1316.

Talairach, J. \& Tournoux, P. (1988) Co-planar stereotaxic atlas of the human brain: 3-dimentional proportional system: An approach to cerebral imaging. Thieme Medical Publishers, Stuttgart.

Thaut, M.H., Demartin, M. \& Sanes, J.N. (2008) Brain networks for integrative rhythm formation. PLoS One, $\mathbf{3}$, e2312.

Van Eimeren, T., Boecker, H., Konkiewitz, E.C., Schwaiger, M., Conrad, B. \& Ceballos-Baumann, A.O. (2001) Right lateralized motor cortex activation during volitional blinking. Ann. Neurol., 49, 813-816.

Wessel, K., Zeffiro, T., Toro, C. \& Hallett, M. (1997) Self-paced versus metronome-paced finger movements. A positron emission tomography study. J. Neuroimaging, 7, 145-151.

Worsley, K.J., Andermann, M., Koulis, T., MacDonald, D. \& Evans, A.C. (1999) Detecting changes in nonisotropic images. Hum. Brain Mapp., 8, 98-101.

Yamamoto, J., Ikeda, A., Satow, T., Matsuhashi, M., Baba, K., Yamane, F., Miyamoto, S., Mihara, T., Hori, T., Taki, W., Hashimoto, N. \& Shibasaki, H. (2004) Human eye fields in the frontal lobe as studied by epicortical recording of movement-related cortical potential. Brain, 127, 873-887. 